\title{
A large close relative of $C$. elegans is slow-developing but not long-lived
}

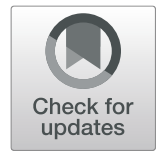

\author{
Gavin C. Woodruff ${ }^{*}$ (D), Erik Johnson and Patrick C. Phillips (D)
}

\begin{abstract}
Background: Variation in body size is thought to be a major driver of a wide variety of ecological and evolutionary patterns, including changes in development, reproduction, and longevity. Additionally, drastic changes in natural context often have profound effects on multiple fitness-related traits. Caenorhabditis inopinata is a recently-discovered fig-associated nematode that is unusually large relative to other members of the genus, including the closely related model system C. elegans. Here we test whether the dramatic increase in body size and shift in ecological context has led to correlated changes in key life history and developmental parameters within this species.

Results: Using four developmental milestones, $C$. inopinata was found to have a slower rate of development than $C$. elegans across a range of temperatures. Despite this, $C$. inopinata did not reveal any differences in adult lifespan from C. elegans after accounting for differences in developmental timing and reproductive mode. C. inopinata fecundity was generally lower than that of C. elegans, but fitness improved under continuous-mating, consistent with spermlimitation under gonochoristic (male/female) reproduction. C. inopinata also revealed greater fecundity and viability at higher temperatures.

Conclusion: Consistent with observations in other ectotherms, slower growth in C. inopinata indicates a potential trade-off between body size and developmental timing, whereas its unchanged lifespan suggests that longevity is largely uncoupled from its increase in body size. Additionally, temperature-dependent patterns of fitness in $C$. inopinata are consistent with its geographic origins in subtropical Okinawa. Overall, these results underscore the extent to which changes in ecological context and body size can shape life history traits.
\end{abstract}

Keywords: Body size, Life history, C. elegans, Lifespan, Heterochrony

\section{Background}

Trade-offs dominate life history evolution. Organisms have access to limited energy resources, and these must be allocated in a balance between self-maintenance and reproductive output. In keeping with the expectation that different distributions of life history traits (such as age of maturity, reproductive duration, and age-specific fecundity, among others) should be sensitive to different distributions of selective pressures on those traits, a huge diversity of patterns among life history traits has emerged across the broad scope of animal diversity [1-5]. As a consequence, many organisms exhibit well-documented correlations among traits such as fecundity and survival [6-8], fecundity and developmental rate [1,9-11], and reproductive quantity and quality $[12,13]$.

\footnotetext{
* Correspondence: gavincw@uoregon.edu

Department of Biology, Institute of Ecology and Evolution, University of Oregon, Eugene, USA
}

Body size is a particularly potent component of life history syndromes. Body size is usually correlated with a multitude of fitness-related traits including developmental rate, offspring number, offspring size, gamete size, and lifespan [14-17]. Body size is also known to covary with physiological traits, such as metabolic rate, thought to underlie trade-offs among life history traits [15, 17]. These factors in turn generate allometric relationships that appear to explain scale-based trends for a wide variety of traits across many orders of magnitude [15]. Indeed, body size appears to be a central component of broad macroevolutionary trends among lineages over geological timescales [18]. But which is cause and which is effect? To what extent does change in body size due to selection on body size per se lead to collected changes in such a wide array of life history traits and to what extent does body size change because of selection acting directly on these traits? 
Life history theory suggests that selection for increased body size can be balanced against the benefits of faster reproduction and the costs of lower offspring viability and lower initial fecundity [1], weighed against a backdrop of differential allocation of physiological and metabolic resources to each of these processes and to growth itself $[17,19]$. At the same time, selection on body size itself must be mediated via environmental factors such as resource availability and/or predation [20]. Although these various causes are not mutually exclusive and likely overlap, the proximate and ultimate causes of body size change-particularly the relationship between these two-remain largely unresolved.

However, body size is not the only factor known to influence life history traits-environmental and ecological change is also expected to promote life history evolution. Indeed, whenever a change in environment impacts the optimal survival and fecundity of different age classes, then life histories will evolve in response [21]. Changes in both abiotic (temperature, salinity, humidity, etc.) and biotic (predation pressure, resource abundance, pollinator density, etc.) environments can impact life history strategies: for instance, differential predation on larval stages in guppies due to spatial differences in predator abundance promoted changes in reproductive effort across populations [21, 22]. Furthermore, the extent of regularity in temporal environments (such as season length) is thought to underlie bet-hedging strategies (such as the germination time decision), and life histories are expected to evolve in response to changes in the timing of environmental cycles as well [21]. Thus, environmental and ecological context plays a critical role in the evolution of life histories. How do changes in ecological context interact with the constraints imposed by body size to promote the evolution of life history traits?

The nematode Caenorhabditis elegans has for decades been an important model for genetics, development, and biology in general [23]. However, the degree and extent of trade-offs between body size and other life history traits in systems like $C$. elegans remain largely unknown and/or have generated somewhat ambiguous or contradictory results [24-32]. Further, because nearly all known members of this genus share a common natural ecological niche of rotting plant material [33], it has not been possible to use a comparative approach to investigate how change in ecological circumstances might drive changes in the relationship between body size and life history [19]. Here, we address this question by taking advantage of a highly phenotypically and ecologically divergent close relative of $C$. elegans: the recently discovered fig-associated nematode C. inopinata.

C. inopinata (formerly known as C. sp. 34 ) is remarkable in that it displays tremendous ecological and phenotypic differences compared to its close relatives [34, 35].
Compared to other Caenorhabditis, C. inopinata is huge: it can grow to be nearly twice as long as other members in the genus $[34,35]$. C. inopinata also develops nearly half as quickly, has sperm three times the size, and embryos $20 \%$ longer than C. elegans [35]. Furthermore, in contrast to the rotting-plant material ecological niche of $C$. elegans and other Caenorhabditis species [36], it thrives in the fresh, intact Okinawan figs of Ficus septica [34, 35, 37]. C. inopinata thus appears to have experienced a radically different selective environment that has led to its highly divergent suite of life history traits. And, as $C$. inopinata is much larger in size and develops much more slowly than its close relatives, it can therefore be used as a natural system to test the predictions of life history theory using a comparative approach. Here, we do just this by describing the developmental timing, lifespan, fecundity, and viability of C. inopinata and C. elegans at multiple temperatures.

\section{Results}

C. inopinata develops more slowly yet does not differ from C. elegans in lifespan and reproductive duration Initial measures of developmental rate revealed that $C$. inopinata develops at about half the rate as C. elegans [35]. To provide a more complete picture of the timing of development in this species, the occurrence of four different developmental milestones (time of hatching, onset of the L4 stage, onset of adulthood, and the onset of reproduction) was ascertained at four different temperatures $\left(15^{\circ} \mathrm{C}, 20^{\circ} \mathrm{C}, 25^{\circ} \mathrm{C}\right.$, and $\left.30^{\circ} \mathrm{C}\right)$ among synchronized populations of $C$. elegans and C. inopinata. Unsurprisingly, all species grew faster as the temperature increased (Fig. 1; Additional file 1: Table S1). Yet in conditions where both species grew reliably, $C$. inopinata was slower to reach all developmental milestones than C. elegans (Fig. 1; Additional file 1: Table S1). Indeed, at the typical rearing temperature of C. elegans $\left(20^{\circ} \mathrm{C}\right)$, the median time of reproductive onset was 2.7 days in C. elegans, whereas it was 6.7 days in C. inopinata (Generalized linear model likelihood ratio test (GLM LRT) chi-square $=4861.4, \mathrm{df}=2, \mathrm{p}<0.0001$ ). To reach a developmental rate that approaches that of $C$. elegans at $20^{\circ} \mathrm{C}, \mathrm{C}$. inopinata must be reared at a temperature that is ten degrees higher (Fig. 1b; Additional file 1: Table S1) where it exhibits reduced fecundity (Fig. 4a) and where C. elegans N2 is inviable (Fig. 5). Overall, then, $C$. inopinata has slower relative growth regardless of temperature.

As slow developing, large animals tend to be longerlived [1], we were curious if $C$. inopinata also exhibits prolonged longevity. To address this, we applied previously established methods of lifespan measurement in nematodes [38] to C. inopinata. As a point of comparison, we also measured C. elegans N2 and C. elegans (fog-2; for Feminization Of Germline) lifespans. As lifespan often 

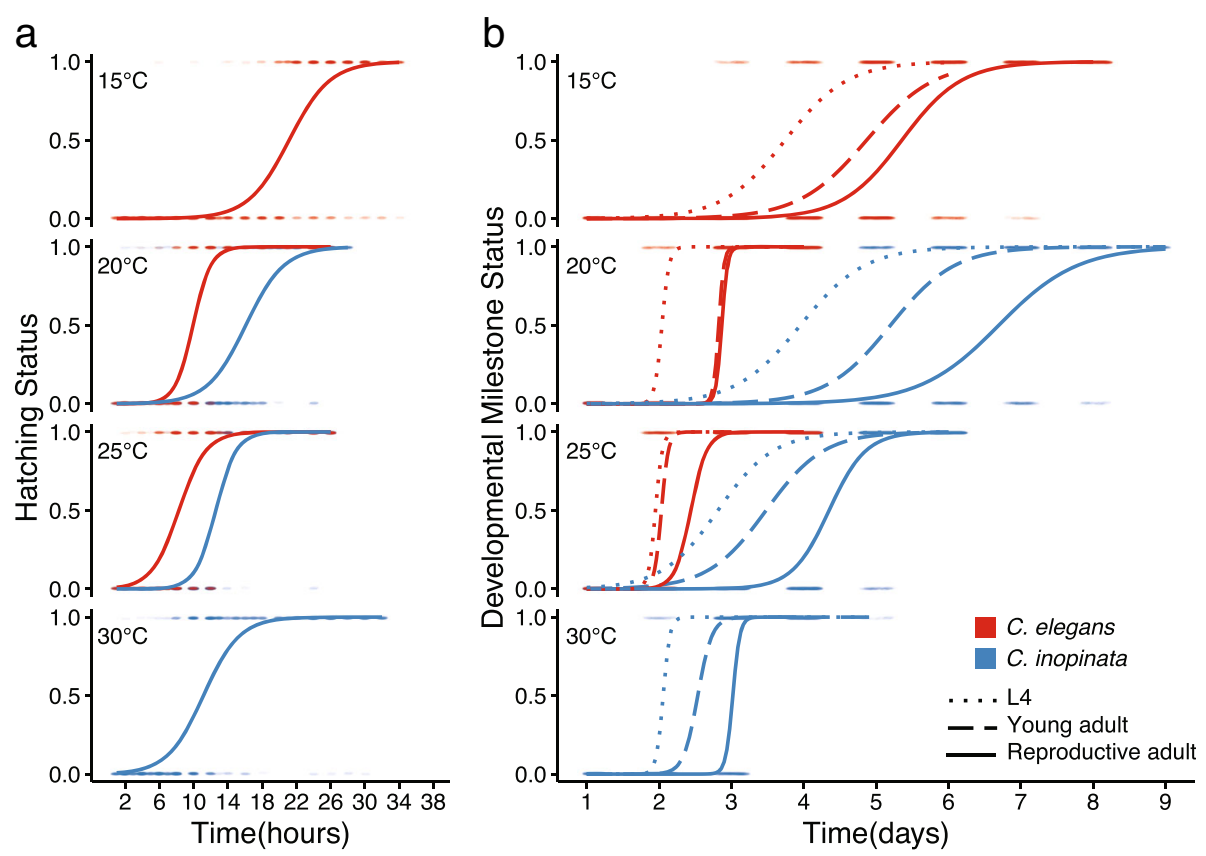

Fig. 1 C. inopinata develops more slowly than C. elegans. The y-axis represents the status of having attained a given developmental milestone; 0 = has not reached milestone, 1 = has reached milestone. Here, the actual data representing animals at (or not at) developmental milestones are plotted as clouds of points at these values over time. The curves are logistic models of growth that were fit to these data (see Additional files 5 and 6 for data and Additional file 4 for software to generate these models). a) Hatching; b) L4, young adulthood, and the onset of reproduction. C. elegans (fog-2) was used for the embryogenesis milestone to account for the delay caused by obligate outcrossing in C. inopinata. C. elegans N2 is inviable at $30^{\circ} \mathrm{C}$, and $\mathrm{C}$. inopinata milestones were not measured at $15^{\circ} \mathrm{C}$ due to its low fitness at this temperature. $\mathrm{N}$ worms $=385$, C. elegans hatching $15^{\circ} \mathrm{C} ; \mathrm{N}=417$, C. inopinata hatching $20^{\circ} \mathrm{C} ; \mathrm{N}=320$, C. elegans hatching $20^{\circ} \mathrm{C} ; \mathrm{N}=383$, C. inopinata hatching $25^{\circ} \mathrm{C} ; \mathrm{N}=319$, C. elegans hatching $25^{\circ} \mathrm{C} ; \mathrm{N}=437, \mathrm{C}$. inopinata hatching $30^{\circ} \mathrm{C} ; \mathrm{N}=225, \mathrm{C}$. elegans $L 415^{\circ} \mathrm{C} ; \mathrm{N}=186, \mathrm{C}$. inopinata $\angle 420^{\circ} \mathrm{C} ; \mathrm{N}=270$, C. elegans $L 420^{\circ} \mathrm{C} ; \mathrm{N}=209, \mathrm{C}$. inopinata $L 425^{\circ} \mathrm{C} ; \mathrm{N}=263, \mathrm{C}$. elegans $L 425^{\circ} \mathrm{C} ; \mathrm{N}=232, \mathrm{C}$. inopinata $\mathrm{L} 430^{\circ} \mathrm{C} ; \mathrm{N}=225$, C. elegans young adult $15^{\circ} \mathrm{C} ; \mathrm{N}=186, \mathrm{C}$. inopinata young adult $20^{\circ} \mathrm{C} ; \mathrm{N}=270$, C. elegans young adult $20^{\circ} \mathrm{C}$; $\mathrm{N}=209$, C. inopinata young adult $25^{\circ} \mathrm{C} ; \mathrm{N}=263, \mathrm{C}$. elegans young adult $25^{\circ} \mathrm{C} ; \mathrm{N}=232$, C. inopinata young adult $30^{\circ} \mathrm{C} ; \mathrm{N}=714, \mathrm{C}$. elegans reproductive adult $15^{\circ} \mathrm{C} ; \mathrm{N}=380, \mathrm{C}$. inopinata reproductive adult $20^{\circ} \mathrm{C} ; \mathrm{N}=677, \mathrm{C}$. elegans reproductive adult $20^{\circ} \mathrm{C} ; \mathrm{N}=784, \mathrm{C}$. inopinata reproductive adult $25^{\circ} \mathrm{C} ; \mathrm{N}=960, \mathrm{C}$. elegans reproductive adult $25^{\circ} \mathrm{C} ; \mathrm{N}=527, \mathrm{C}$. inopinata reproductive adult $30^{\circ} \mathrm{C}$. GLM LRT chi-square $\mathrm{p}<0.0001$ for every C. elegans and C. inopinata comparison.

trades-off with reproductive output [39, 40], we used virgin C. elegans (fog-2) pseudo-females (which do not generate self-sperm and are self-sterile as a consequence [41]) to control for differences in reproductive mode. C. inopinata females were longer-lived than wild-type C. elegans hermaphrodites at $25^{\circ} \mathrm{C}$, with a median total lifespan that was four days higher $(20$ and 16, respectively; Cox proportional hazards linear model comparison, $\mathrm{Z}$-value $=4.99, \mathrm{p}<0.0001$ Fig. $2 \mathrm{a}$; Additional file 1: Figure S1). However, C. inopinata females were only marginally longer lived than C. elegans (fog-2) pseudo-females (19 days, Cox proportional hazards linear model comparison, $\mathrm{Z}$-value $=2.29, \mathrm{p}=0.053$ ). Furthermore, no differences in adult lifespan (which takes into account the differences in developmental timing between $C$. elegans and C. inopinata) were detected between $C$. inopinata females (median adult lifespan of 16 days) and C. elegans (fog-2) pseudo-females (median adult lifespan of 17 days; Cox proportional hazards linear model comparison, $\mathrm{Z}$-value $=0.74, \mathrm{p}=0.73$; Fig. $2 \mathrm{~b}$; Additional file 1: Figure S2). Thus, despite its large size and slow development, $C$. inopinata adults are not longer-lived than C. elegans after accounting for differences in reproductive mode and developmental timing.

The duration of reproduction is also expected to trade-off with growth rate and body size [1, 2], with large, slow-developing animals tending to have longer reproductive periods [9-11]. To see if this also holds for C. inopinata, daily measures of fecundity were made with individual C. elegans (fog-2) pseudo-females and C. inopinata females under conditions of continuous mating throughout their lifetimes (Fig. 3). Although one individual $C$. inopinata female had a reproductive duration of twelve days, for the most part, both species lay almost all of their embryos in the first four days of adulthood (Fig. 3b). Indeed, under continuous mating conditions at 

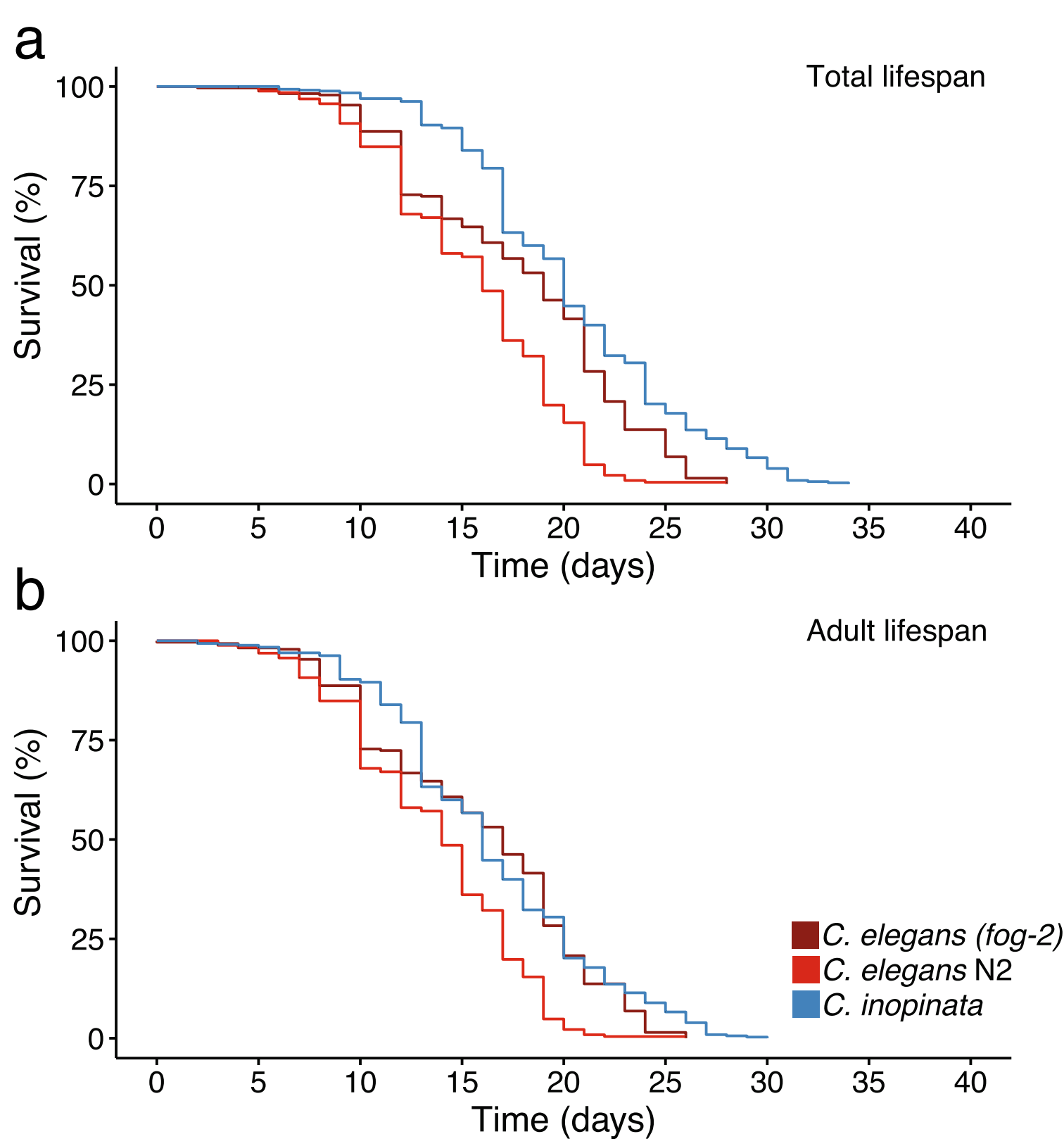

Fig. 2 C. inopinata is not longer-lived than C. elegans at $25^{\circ} \mathrm{C}$ after taking reproductive mode and developmental timing into account. (a) Total lifespan models. Here, Day $=0$ represents the day embryos were laid. (b) Adult lifespan models. Here, Day $=0$ is the approximate first day of adulthood, taken as the total lifespan minus two (C. elegans) or four (C. inopinata) days. Wild-type C. elegans N2 exhibits both shorter total and adult median lifespan than C. inopinata. Conversely, C. inopinata females have a marginally higher median total lifespan than non-selfing C. elegans (fog-2) mutant females, and no difference in C. inopinata and C. elegans (fog-2) adult lifespan was detected (Cox proportional hazards linear model comparison, Z-value $=0.74, \mathrm{p}=0.73$ ). $\mathrm{N}$ worms=263 (C. elegans N2), N=281 (C. elegans (fog-2)), N=444 (C. inopinata).

$25^{\circ} \mathrm{C}$, no differences in brood fraction per day could be detected between $C$. inopinata and $C$. elegans with the exception of day eight of adulthood (Wilcoxon rank sum test, $\mathrm{W}=528, \mathrm{p}=0.041$ ). Thus, like lifespan, duration of reproduction is not extended in C. inopinata.
C. inopinata is sperm-limited and reveals higher fitness at higher temperatures

Brood size also tends to covary with both body size and developmental rate $[1,2]$, and so fecundity was measured at four different temperatures in $C$. inopinata and $C$. 


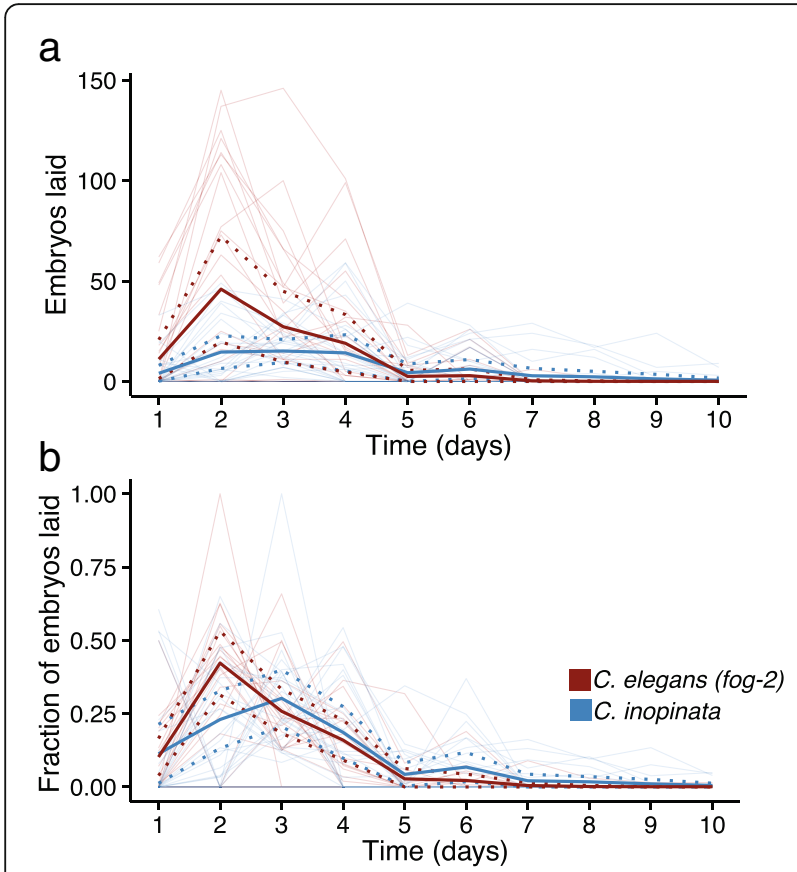

Fig. 3 C. inopinata has a reproductive duration comparable to $C$. elegans. (a) Number of embryos laid per day. (b) Fraction of lifetime brood laid per day. Bold lines represent averages, and dotted bold lines represent \pm 1 SDM. Thin lines represent individual worms. The $C$. elegans (fog-2) and C. inopinata day two and three brood fractions are not statistically different (Wilcoxon rank sum test $W=389 p=0.36$ and $\mathrm{W}=553 \mathrm{p}=0.13$, respectively). $\mathrm{N}$ parental females $=30$ for both species. All observations taken at $25^{\circ} \mathrm{C}$.

elegans (fog-2) to address if similar patterns hold in this group (Fig. 4). In conditions in which females were mated with males for just one night, $C$. inopinata generally displayed far smaller brood sizes than C. elegans (fog-2), with the exception that C. elegans $(f \circ g-2)$ is infertile at $30^{\circ} \mathrm{C}$ (Fig. 4a). However, as the male/female species C. remanei is known to generate more progeny when constantly exposed to males $[42,43]$, we suspected that $C$. inopinata might also be sperm-limited. Indeed, under continuous mating conditions, there is no detectable difference in brood size between C. inopinata and C. elegans (fog-2) (median brood size of 58 and 76, respectively; Wilcoxon rank sum test, $W=484 \mathrm{p}=0.62$; Fig. $4 \mathrm{~b}$ ). However, male mating performance tends to degrade in selfing species [44], so we also compared the fraction of successful crosses between C. elegans and C. inopinata (Additional file 1: Figure S3). In continuous mating conditions, the fraction of failed crosses was higher in C. elegans $(0.33, \mathrm{~N}=30$ crosses) than in C. inopinata (0.17, $\mathrm{N}=30$ crosses), although this difference was not statistically significant (Fisher's Exact Test odds ratio $=2.46, \mathrm{p}=0.23$ ). After removing animals that failed to produce progeny, C. elegans (fog-2) yielded a median brood size that is over twice as large as that of C. inopinata in continuous mating conditions (145 and 65, respectively; Wilcoxon rank sum test, $\mathrm{W}=359$, $\mathrm{p}=0.013$; Additional file 1: Figure S4). Thus C. inopinata requires constant access to mates in order to maximize its reproductive output, consistent with its gonochoristic mode of reproduction.

When examining the relationship between developmental rate and fecundity, the intrinsic rate of increase $(r)$ is likely a better measure of fitness than total fecundity $\left(R_{0}\right)[1,45]$. Under this approach, fitness is a function of age-specific fecundity and viability, and the age of first reproduction can highly influence the population growth rate [1]. So although $C$. inopinata and $C$. elegans have comparable brood sizes under continuous mating conditions, they likely differ in fitness because of their different developmental rates. Indeed, despite their comparable brood sizes, C. elegans has a rate of increase $(r=1.54,95 \%$ confidence interval $(\mathrm{CI})=1.26-1.72)$ that is over twice as high as C. inopinata $(r=0.66,95 \% \mathrm{CI}=0.54-0.74)$. This difference in fitness is even greater in mating conditions with just overnight access to males (C. elegans $r=2.09$, 95\% $\mathrm{CI}=1.88-2.24 ; C$. inopinata $r=0.63,95 \% \mathrm{CI}=0.55-0.69)$. Thus continuous access to males is not sufficient to overcome the detriment to fitness due to slow development in C. inopinata.

In keeping with the other life-history measures, $C$. elegans was more viable at lower temperatures and $C$. inopinata more viable at higher temperatures during early development (Fig. 5). Overall, however, C. inopinata displayed consistently lower embryo-to-adult viability than C. elegans at $15^{\circ} \mathrm{C}, 20^{\circ} \mathrm{C}$, and $25^{\circ} \mathrm{C}$ (Wilcoxon rank sum test $\mathrm{p}<0.001$ in all comparisons; Fig. 5). No detectable differences in $C$. inopinata viability were found between $20^{\circ} \mathrm{C}, 25^{\circ} \mathrm{C}$, and $30^{\circ} \mathrm{C}$ (median viability of $0.84,0.79$, and 0.88 , respectively; Wilcoxon rank sum test $\mathrm{W}=50 \mathrm{p}=0.060$, $\mathrm{W}=70 \mathrm{p}=0.62$; Fig.5), but $C$. inopinata is less viable at $15^{\circ} \mathrm{C}$ (median viability of 0.63 ; Wilcoxon rank sum test $\mathrm{p} \leq 0.030$ for all comparisons). As C. inopinata fecundity is also higher at warmer temperatures (Fig. 4a), these temperature-specific fitness patterns are consistent with its subtropical natural context of fresh Okinawan Ficus septica figs.

\section{Most C. elegans genes with life history phenotypes conventionally associated with large body size intersect with only one phenotype}

Life history syndromes are often thought to be driven by trade-offs resulting from antagonistic pleiotropy [46]. How often are pleiotropic effects observed in life history traits in C. elegans? The C. elegans genomic database WormBase [47] has collected gene-specific information regarding the biological consequences of mutation and RNAi exposure as "phenotype" terms, which constitute a formal ontology used to describe phenotypes associated 

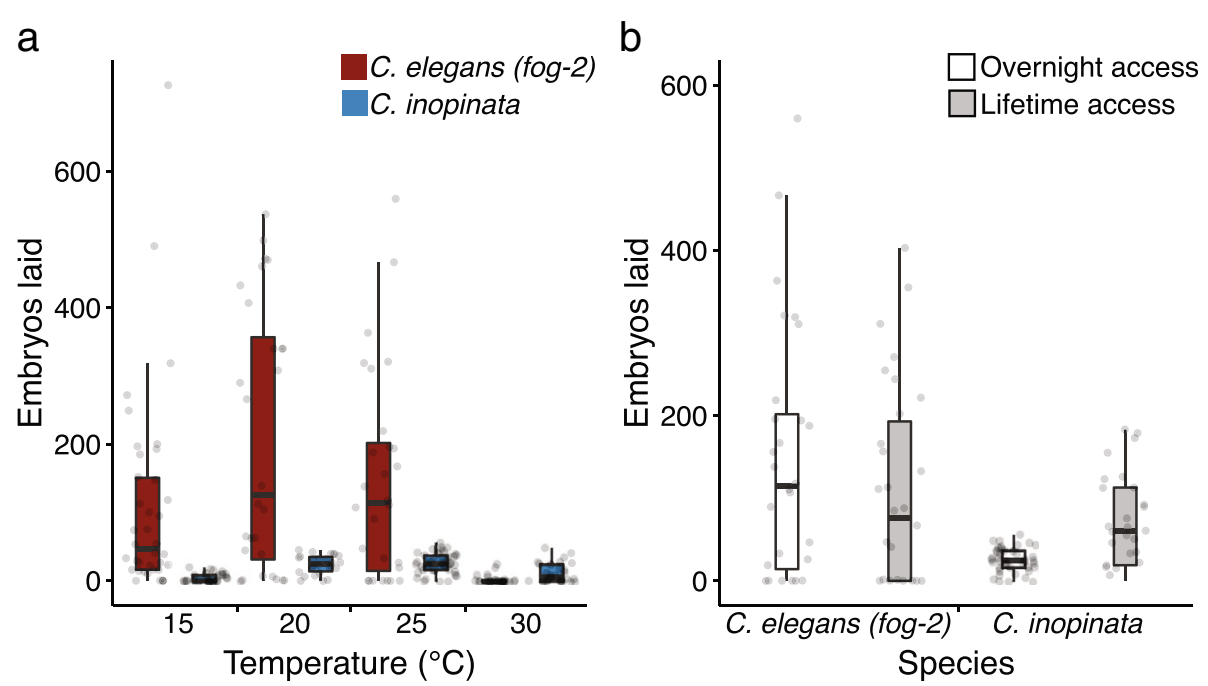

Fig. 4 C. inopinata is sperm-limited. (a) Number of embryos laid in single overnight mating conditions at various temperatures. (b) Number of embryos laid in continuous mating or single overnight mating conditions at $25^{\circ} \mathrm{C}$. The "one overnight mating" data in panel (b) is the same from those at $25^{\circ} \mathrm{C}$ in panel (a). C. inopinata has smaller broods than C. elegans (fog-2) in every condition except $30^{\circ} \mathrm{C}$ (Wilcoxon rank sum test $\mathrm{p}<0.0001$ for 15 and $20^{\circ} \mathrm{C} ; \mathrm{W}=349, \mathrm{p}=0.004$ for $25^{\circ} \mathrm{C} ; \mathrm{W}=575, \mathrm{p}=0.002$ for $30^{\circ} \mathrm{C}$ ). However, there is no detectable difference in $\mathrm{C}$. elegans (fog-2) and C. inopinata brood sizes under continuous mating conditions (Wilcoxon rank sum test, $\mathrm{W}=484, \mathrm{p}=0.62$ ). $\mathrm{N}$ parental females $=38, \mathrm{C}$. elegans overnight access $15^{\circ} \mathrm{C}$; $\mathrm{N}=28, \mathrm{C}$. inopinata overnight access $15^{\circ} \mathrm{C} ; \mathrm{N}=28$, C. elegans overnight access $20^{\circ} \mathrm{C} ; \mathrm{N}=26, \mathrm{C}$. inopinata overnight access $20^{\circ} \mathrm{C} ; \mathrm{N}=28, \mathrm{C}$. elegans overnight access $25^{\circ} \mathrm{C} ; \mathrm{N}=42$, C. inopinata overnight access $25^{\circ} \mathrm{C} ; \mathrm{N}=28$, C. elegans overnight access $30^{\circ} \mathrm{C} ; \mathrm{N}=28, \mathrm{C}$. inopinata overnight access $30^{\circ} \mathrm{C} ; \mathrm{N}=30, \mathrm{C}$. elegans lifetime access $25^{\circ} \mathrm{C} ; \mathrm{N}=30$, C. inopinata lifetime access $25^{\circ} \mathrm{C}$.

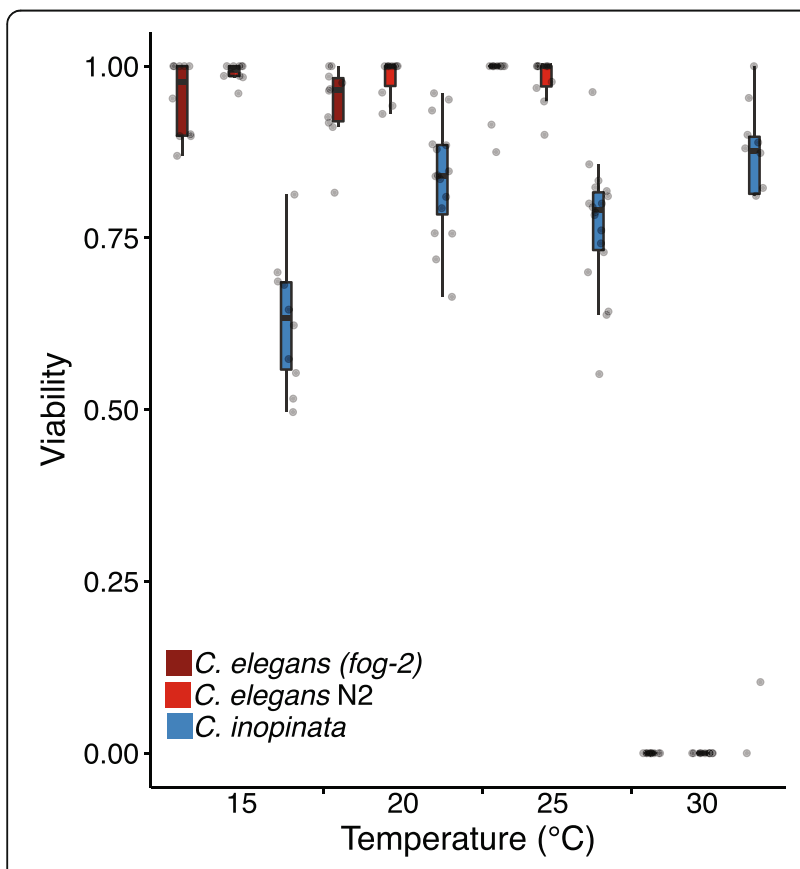

Fig. 5 C. inopinata has a lower viability than C. elegans. Embryo-toadult viability at four temperatures. C. elegans reveals higher viability in all conditions except $30^{\circ} \mathrm{C}$ regardless of reproductive mode. $\mathrm{N}$ plates $=10$, C. inopinata $15^{\circ} \mathrm{C} ; \mathrm{N}=10$, C. elegans $\mathrm{N} 215^{\circ} \mathrm{C} ; \mathrm{N}=10$, C. elegans (fog-2) $15^{\circ} \mathrm{C} ; \mathrm{N}=16, \mathrm{C}$. inopinata $20^{\circ} \mathrm{C} ; \mathrm{N}=10$, C. elegans $\mathrm{N} 220^{\circ} \mathrm{C} ; \mathrm{N}=10, \mathrm{C}$. elegans (fog-2) $20^{\circ} \mathrm{C} ; \mathrm{N}=18$, C. inopinata $25^{\circ} \mathrm{C} ; \mathrm{N}=10$, C. elegans $\mathrm{N} 225^{\circ} \mathrm{C}$; $\mathrm{N}=10$, C. elegans (fog-2) $25^{\circ} \mathrm{C} ; \mathrm{N}=10, \mathrm{C}$. inopinata $30^{\circ} \mathrm{C} ; \mathrm{N}=10$, C. elegans $\mathrm{N} 230^{\circ} \mathrm{C} ; \mathrm{N}=10$, C. elegans (fog-2) $30^{\circ} \mathrm{C} ; \mathrm{N}$ embryos per plate $=5-237$. with genes [48]. To explore the extent of pleiotropy underlying life history syndromes in C. elegans, we measured the amount of overlap among four WormBase database phenotypes that resemble life history traits associated with large body size ("long," "extended life span," "reduced brood size," and "slow growth") in $C$. elegans protein-coding genes (Fig. 6). As previously shown [49], most C. elegans protein-coding genes do not have any reported phenotypes $(42 \%$ or $8,585 / 20,209)$. $14 \%$ of C. elegans protein-coding genes $(2,908 / 20,209)$ had at least one of the four life history phenotypes. Of these, the vast majority $(74 \%$ or $2,159 / 2,908)$ intersected with only one of the four phenotypes (Fig. 6). This suggests that these traits are potentially largely genetically decoupled in this system and that pleiotropy need not underlie the evolution of life history strategies.

\section{Discussion}

Possibly because it is both obvious and easy to measure, body size variation has been studied extensively for centuries. The range in body size across the tree of life is so immense as to demand explanation (21 orders of magnitude $[16,50])$, and this incredible diversity has spawned a vast and rich literature attempting to comprehend its origins and maintenance. One major conclusion from this research program is that body size is correlated with nearly every trait, such that long-established relationships between body size and growth, reproduction, and lifespan underscore a prominent role for body size in 


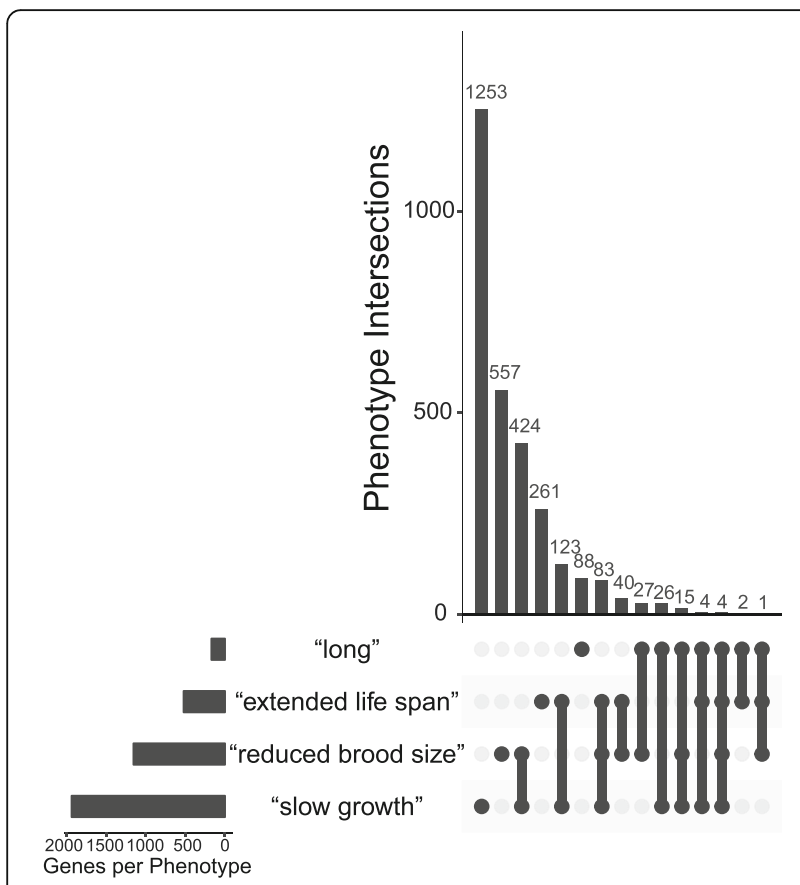

Fig. 6 Intersection of relevant life history trait phenotypes in C. elegans protein-coding genes. In C. elegans, many genes that can increase body length, slow development, extend lifespan, or reduce fecundity when defective do not also promote correlated changes in life history traits often associated with increased body size. Matrix layout plot shows intersections of $C$. elegans genes among four WormBase phenotype terms [48] ("long," "extended life span," "reduced brood size," "slow growth"; plot generated in R with the UpSetR package [86]). Most genes intersect with only one phenotype (the first, second, fourth, and sixth columns from the left), whereas only four genes display all four phenotypes. Most protein-coding genes in C. elegans do not have any reported phenotypes (also see analysis in [49]). See methods for more details.

the evolution of life histories $[14,15,50]$. Here, we found that an exceptionally large and ecologically divergent close relative of C. elegans exhibits slow growth and low fecundity across a range of temperatures yet is not long lived. Together with the extensive C. elegans literature and the foundations of life history theory, these observations can contribute to our understanding of the causes and consequences of large-scale changes in body size and ecological divergence.

\section{The impact of ecological divergence on life history syndromes}

Changes in ecological context are expected to impact life history traits. Here, we described the life history traits of C. inopinata, an organism that occupies an exceptional ecological niche when compared with its close relatives. Most Caenorhabditis species, including C. elegans, thrive in rotting plant material [36]. C. inopinata proliferates in fresh $F$. septica figs, living in close association with its pollinating fig wasps [34, 37]. How might this dramatic shift in ecological context explain the patterns of life history traits observed here?

C. inopinata grows at nearly half the rate as C. elegans (Fig. 1). One possible explanation for the divergence in developmental rate could be its novel natural context. Most Caenorhabditis proliferate in the ephemeral environments of rotting vegetation [36]; it is thought that the rapid turnover and spatial patchiness of its bacterial food resources has driven its rapid development, high fecundity, and its resource-dependent decision to enter the dispersal dauer larval stage [51]. C. inopinata, conversely, grows in the presumably more stable environment of the fig lumen and obligately disperses on pollinating fig wasps $[34,37]$. Figs generally take weeks to develop [52], although it is unclear how many generations of worms occur within a single fig. It is then possible that the extreme divergence in developmental rate might be connected to differences in the transience of resource availability between these environments. Furthermore, as $C$. inopinata animals disperse to new figs via pollinating wasps [37], their life cycle is necessarily closely tied to patterns of wasp development and emergence, consistent with correlations among Ceratosolen fig wasp and $C$. inopinata developmental stages that have been found in previous field studies [37]. This contrasts with $C$. elegans and its other close relatives, who disperse on a plethora of invertebrate carriers (including isopods, myriapods, and gastropods) [53]. Future longitudinal field studies of single fig trees at finer temporal resolution will be required to determine the relative paces of fig, fig wasp, and nematode development in nature and to test hypotheses regarding the ecological drivers of heterochrony.

Overall, C. inopinata reveals a lower viability (Fig. 5) and fecundity (Fig. 4) than C. elegans in laboratory conditions, although lifetime access to males greatly improves C. inopinata fecundity (Fig. 4b). How might its unique ecology underlie these patterns? A particularly interesting avenue to pursue is based on the observation that wild bacteria associated with Caenorhabditis can have both positive or negative influences on fecundity and growth $[54,55]$ and that different species of Caenorhabditis are associated with different microbes in nature [54]. Thus the nutritional environment can have a profound effect on fitness and life history traits. The natural microbial food of C. inopinata is currently unknown. As C. inopinata exhibits reduced gonads in laboratory culture [35], it may be experiencing nutritional deficiencies. The reduced fecundity of $C$. inopinata may then reflect a plastic response to an adverse environment as opposed to a trade-off with increased body size. The potential influence of natural microbial associates of Ficus septica figs on C. inopinata fitness affords an exciting opportunity for future research. 
The large body size of $C$. inopinata could also be more directly connected to its novel environmental context. Body size is broadly correlated with fecundity across nematode phylogeny [56-58], and the fig microcosm may represent an environment with less selective pressure on body size than rotting vegetation. Hence $C$. inopinata may be large because its ecological context reveals relaxed selection on body size compared to its close relatives. Such pressures may entail predation or pathogens-various mites, fungi, bacteria, and viruses are known to prey on or infect Caenorhabditis [53]. Figs maintain many defenses against antagonistic invertebrates and microorganisms [59-61], and it is likely that this environment harbors a less diverse community than rotting vegetation. In this case any trade-offs among predation or infection avoidance and body size would be lifted, facilitating body size change resulting from selection on increased fecundity. However, within C. elegans, no evidence for correlations between body size and fecundity was found after artificial selection for large body size [25]. Thus it is entirely possible that such trade-offs are not driving body size change in this case and that other factors (adaptive or not) are responsible upon shifting to the fig environment. Further studies explicitly addressing the possible ecological determinants of body size change will be needed to delineate these possibilities.

\section{Developmental timing and body size}

It makes intuitive sense that larger organisms should develop more slowly. Being more massive, presumably more cell divisions and/or biosynthetic reactions must take place for their construction and it therefore follows that their development should take longer than smaller organisms. And this intuition bears out across vast phylogenetic distances: from bacteria to sequoias, body size covaries with generation time [50]. Here, we found that in all temperatures, $C$. inopinata grows nearly half as quickly as C. elegans, consistent with previous observations (Fig. 1; [34, 35]). Indeed, $C$. inopinata needs to be grown at $30^{\circ} \mathrm{C}$ to approach a rate of development comparable to that of $C$. elegans when grown at $20^{\circ} \mathrm{C}$. Thus, the observation that this very large species also develops much more slowly than its close relatives is in line with decades of allometric studies. Further, as cell size is coordinated with cell division decisions in multiple organisms $[62,63]$, body size change could occur even in the absence of cell number change through the modification of cell cycle timing. This may explain the case of $C$. inopinata, as previous observations observed no change in cell number despite its large size and slow development [35].

However, there are reasons to suspect slow development may not underlie large body size in this case. It has been argued that the allometric trends observed in birds and mammals cannot be easily extended to poikilotherms because of difficulties in comparing physiological time due to rapid change in metabolic rates [16]. More notable is the common observation that developmental timing can be decoupled from body size in C. elegans. Most mutations in C. elegans that extend body length do not also slow the rate of growth: only $29 \%$ of the genes in the $C$. elegans genome known to control body length also promote slower development (Fig. 6). Furthermore, experimental evolution and mutation accumulation studies in C. elegans and C. briggsae have not generally reported correlated changes in body size and developmental timing $[25,27,28,64]$. Thus, it appears that body size and rate of growth need not be strongly coupled in Caenorhabditis and that the relationship between these traits observed in C. inopinata may not necessarily be causative. Furthermore, as mentioned above, the slow growth of C. inopinata may also be better understood with respect to its natural ecological context (or could also be due to nutritional deficiencies in a laboratory context); framing the causes of heterochronic change with respect to this context is an exciting avenue for future studies.

\section{Reproduction and body size}

The relationship between body size and reproduction varies both within and between taxa. In birds and mammals, larger species tend to have lower fecundities than smaller species [15]. Conversely, body size appears to be positively correlated with fecundity in insects [65] and nematodes [56-58]. C. inopinata was generally found to have lower brood sizes than $C$. elegans across a range of temperatures (Fig. 4a), although continuous mating greatly improves fecundity in C. inopinata (Fig. 4b). The relatively low fecundity of $C$. inopinata is then incongruent with patterns of fecundity and body size that have been previously reported among nematodes [56-58]. C. inopinata's gonochoristic mode of development cannot explain its low brood size, as multiple male/female species of Caenorhabditis have been reported to have higher brood sizes [42, 43, 66-69]. However, the sperm-limited fecundity of $C$. inopinata (Fig. 4b) is consistent with previous observations with the gonochoristic C. remanei $[42,43]$. It is possible that the evolution of extreme body size in the case of $C$. inopinata reveals a trade-off with reproductive output, wherein resources usually allocated to progeny have instead been shifted to increase self-maintenance and growth. Yet most genes known to regulate body length in the $C$. elegans genome do not appear to have a pleiotropic role in brood size (only $28 \%$ do; Fig. 6). This is also consistent with experimental evolution studies in Caenorhabditis [25], wherein fecundity and body size do not necessarily trade-off. So again, the precise causal relationship here bears further study. Additionally, as mentioned above, patterns of fecundity in C. inopinata may 
also be better understood with respect to its natural environment. It is possible that $C$. inopinata may be experiencing nutritional deficiencies in the laboratory context, and future studies with natural associated microbes will help to inform this possibility.

\section{Lifespan and body size}

Lifespan is often positively correlated with body size, and from an allometric perspective is usually thought to be regulated by variation in developmental and metabolic rates $[15,17]$. And although the age of maturity is sensitive to selection under a range of trait distributions in life history theory [1], from an evolutionary perspective it is thought that late-life traits are generally not subject to selection as its strength falls to zero once reproduction ends [3]. Despite its large size and slow development, $C$. inopinata was found to have only a marginally longer lifespan than C. elegans (Fig. 2). And, when differences in developmental timing and reproductive mode are taken into account, C. inopinata adult lifespan is not significantly different from that of C. elegans (Fig. 2b). The lack of lifespan change in this system is consistent with the view that lifespan is under weak selection, as $C$. inopinata has experienced dramatic change in many other traits under its novel ecological context [34, 35, 37]. Indeed, most lifespan-extending mutations identified in C. elegans have not been associated with pleiotropic effects on body size (Fig. 6). Similarly, experimental evolution studies in C. elegans show no correlated responses in lifespan upon artificial selection on early fecundity [32] and body size [25]. Additionally, no relationships between lifespan and fecundity have been found in mutation-accumulation lines [24] or among wild isolates [26]. These observations are inconsistent with the antagonistic pleiotropy explanation of aging, which posits that the greater fitness contribution of early life survival and reproduction leads to late life deterioration because of negative genetic correlations of these traits [70]. Rather, lifespan appears to be possibly largely uncoupled from fitness-related traits in this group, consistent with the unchanged longevity observed in C. inopinata. However, the nutritional caveats in this system noted in the above interpretation of observed patterns of fecundity also apply here. It is possible that $C$. inopinata will be longer-lived under different rearing conditions, and measurements of lifespan of $C$. inopinata raised on bacterial food originating from its natural context need to be performed.

\section{Variation, inbreeding, and fitness-related traits}

The observations reported here constitute comparisons of two species with each represented by one genetic strain. How does this fact impact the implications of this work discussed here? From a comparative phylogenetics perspective, this sample size is simply insufficient to make broad generalizations about patterns of life history trait covariation in the Caenorhabditis genus. It remains possible that $C$. inopinata represents an outlier that defies meaningful biological trends that we would otherwise not capture because of our limited phylogenetic sample. Furthermore, as we have only interrogated one strain of C. inopinata, this strain may also not be representative of this species as such. Ultimately, broad sampling and measures across Caenorhabditis phylogeny will be needed to make solid claims along these lines. However, can previous observations of variation in fitness-related traits in Caenorhabditis help to overcome this limitation or better inform these results?

C. inopinata is an exceptional Caenorhabditis species with respect to its large body size [34, 35], and most members of the Elegans group are difficult to distinguish morphologically [71, 72]. But, as mentioned above, there is variation both within and between Caenorhabditis species in fecundity (Additional file 1: Figures S5-S6; Additional file 2) and developmental rate (Additional file 1: Figures S7-S8; Additional file 3). Among four recent studies that measured fecundity in Caenorhabditis (including this study) [38, 42, 73] , which includes 24 strains among four species, our estimate of C. inopinata fecundity at $20^{\circ} \mathrm{C}$ is the lowest (Additional file 1: Figures S5-S6). But as C. inopinata and other gonochoristic Caenorhabditis are sperm-limited (Fig. 4) [42, 43], this is likely an underestimate of its reproductive capacity. However, even limited access to males can maintain brood sizes in C. remanei wild isolates that exceed those observed in many strains of selfing species (Additional file 1: Figures S5-S6) [42], including C. elegans $\mathrm{N} 2$, which is considered a domesticated laboratory strain [74]. With respect to previous studies of developmental timing, $C$. inopinata is even more extreme in its divergence from its close relatives, developing at about half the rate as the next slowest strain (C. tropicalis JU1630) among those considered in two previous publications (Additional file 1: Figures S7-S8) [38, 75]. Thus a broader phylogenetic context with more Caenorhabditis species and strains also suggests that this $C$. inopinata strain harbors an exceptionally slow developmental rate and a low (but sperm-limited) fecundity in addition to its large body size.

Gonochoristic Caenorhabditis species are susceptible to inbreeding depression [76, 77]. Could C. inopinata strain NKZ2 have experienced inbreeding depression during laboratory culture that impacts estimates of fitness-related traits? This possibility cannot be definitively ruled out, and an important caveat of these results is that inbreeding depression may influence the patterns of life history traits observed here. However, there are reasons to suspect inbreeding depression may not be a major influencing factor in this case. Primarily, 
C. inopinata NKZ2 is a wild isolate that has not been deliberately inbred, and it is a strain derived from multiple founding individuals [35]. If inbreeding depression were the only cause of declines in fitness-related traits, then C. inopinata NKZ2 should be expected to have higher fecundity than isofemale wild isolates and inbred lines of other gonochoristic species as it is derived from more than one founding individual. As this is not the case (Additional file 1: Figures S5-S6), this suggests that inbreeding depression alone may not be driving these patterns. Furthermore, the ecology of $C$. inopinata may render it less susceptible to inbreeding depression through its obligate dispersal on fig wasps [37]. Despite this, the embryo-to-adult viability of $C$. inopinata is low across multiple temperatures (Fig. 5), which is itself suggestive of inbreeding depression. As we did not measure larval survival directly, it is unclear at what stage animals are undergoing developmental arrest. Additionally, it is possible that behavior could also be driving these results-larvae that crawl off plates and do not mature will artificially deflate viability measurements. However, as dead embryos have been anecdotally observed in these cultures, embryonic lethality is likely driving at least some of these patterns. It also remains possible that the culture conditions designed for C. elegans are insufficient for the reliable development of this species, and native fig, wasp, or microbial factors may be needed for robust $C$. inopinata viability. Regardless, future studies that rear this species in more ecologically-relevant culture conditions, use $C$. inopinata inbred lines and wild isolates, and implement population genomic approaches in natural populations will be needed to disentangle these possibilities.

\section{Pleiotropy and life history syndromes}

Here we note relationships among life history traits across two species of Caenorhabditis. What role does pleiotropy play in the patterns observed here, and are life history syndromes the result of indirect selection, direct selection on multiple characters, or both? Because this study does not directly interrogate the genetic basis of these traits, the underlying genetic causes of these relationships are undetermined. However, the vast background information associated with the $C$. elegans model system can provide context to generate hypotheses regarding the evolution of life history strategies. How many genes have pleiotropic effects on multiple life history traits in C. elegans? As discussed above, most genes associated with one of four life history WormBase phenotypes [48] that might be associated with large body size ("slow growth", "reduced brood size," "extended life span," and "long") do not intersect with one another (Fig. 6). That is, most genes with any of these phenotypes are associated with only one of the four (74\%; Fig. 6), and only a fraction of them reveal evidence of pleiotropic effects
(26\%; Fig. 6). Thus these life history traits appear to be largely genetically decoupled in this group, and pleiotropy need not underlie the correlated evolution of these traits. However, as some genes do influence multiple phenotypes (Fig. 6), pleiotropy may still contribute to the evolution of life history syndromes in this case. Furthermore, as many $C$. elegans studies are largely concerned with only a few phenotypic traits of interest, these results are likely to underestimate the extent of pleiotropy among C. elegans genes. Regardless, future work investigating the genetic bases of these traits in C. inopinata will be needed to understand the role of pleiotropy in shaping life history syndromes.

\section{Temperature-dependent patterns of fitness-related traits in C. Inopinata}

Notably, C. inopinata was more fit at higher than lower temperatures (Fig.4a, Fig. 5). Temperature-dependent plasticity of fitness-related traits varies both within and between species in Caenorhabditis, and these patterns often coincide with ecological context. Within C. briggsae, there are definable clades that are genetically structured by latitude $[78,79]$, and these wild isolates reveal temperaturedependent patterns of fecundity that are consistent with their geographical origin [80]. Additionally, the tropical species C. nigoni [66, 81] and C. tropicalis [82] have higher fitness at warmer temperatures. As C. inopinata has only been found in the subtropical islands of Okinawa [34, 35], its temperature-dependent patterns of fitness are consistent with these previous observations. And further, the temperatures where $C$. inopinata has shown the highest fitness here are comparable to natural Ficus septica fig temperatures measured in nature [37]. Additionally, C. inopinata needs to be grown at $30^{\circ} \mathrm{C}$ to approach a rate of development comparable to that of C. elegans when grown at $20^{\circ} \mathrm{C}$. Could the slow growth of $C$. inopinata more appropriately be interpreted as an adjustment of optimal developmental timing imposed by its subtropical environment? This explanation is appealing as thermal plasticity in growth is widespread in ectotherms, and wild $F$. septica fig interiors were found to harbor temperatures of $29^{\circ} \mathrm{C}$ on average [37]. However, if temperature were a major driver of a universal, optimal developmental rate in Caenorhabditis, then we would expect to see much slower development in tropical strains and species than has been reported (Additional file 1: Figure S8). Indeed, there are no detectable developmental timing differences between tropical and temperate strains of C. briggsae (Additional file 1: Figure S8) [38], which nonetheless do reveal clade-specific, temperature-dependent differences in fecundity [80]. And although C. tropicalis, which is typically found in warmer climates than C. inopinata [51, 83], harbors a slower developmental rate than $C$. elegans (Additional file 1: Figure S7-S8) [38], it remains far faster than that of C. inopinata 
(Additional file 1: Figure S7-S8). Thus, it seems more likely that the slow growth of $C$. inopinata is connected to its novel ecological context in $F$. septica figs, its exceptionally large body size, or both, rather than its subtropical locality alone. Regardless, as a close relative of C. elegans, this species is well positioned for uncovering the genomic bases of temperature adaptation.

\section{Conclusions}

Body size and ecological divergence are major drivers of evolutionary change in multiple taxa, and such changes often co-occur with widespread change in life history traits. Here, we examined the life history traits of a large, ecologicallydivergent close relative of $C$. elegans. We found that C. inopinata develops at nearly half the rate as C. elegans, revealing a likely trade-off between growth and body size. Conversely, longevity does not evolve as part of correlated response to selection on body size in this system, consistent with previous studies and indicative of genetic decoupling of longevity from other life-history traits. Future studies that situate these systems within their natural ecological contexts will be needed to fully disentangle matters of cause and effect among the traits that constitute life history strategies. Taken together, these observations reveal that drastic change in ecological context and body size do not necessarily have an allencompassing impact on life history syndromes.

\section{Methods}

\section{Strains and maintenance}

Animals were maintained on Nematode Growth Media (with 3.2\% agar to discourage burrowing) supplemented with Escherichia coli strain OP50-1 for food. The C. inopinata wild isolate strain NKZ2 [35] was utilized for all observations in this report. C. elegans $\mathrm{N} 2$ and the obligate outcrossing C. elegans fog-2(q71) JK574 [41] mutant strain were also used for most comparisons. Notably, C. elegans is hermaphroditic, while C. inopinata is male/female or gonochoristic. This makes interspecific comparisons problematic. Thus the fog-2(q71) mutation, which prevents spermatogenesis only in hermaphrodites but promotes no obvious somatic defects in either sex [41], was used to control for differences in reproductive mode in various comparisons of life history traits.

\section{Developmental timing}

The timing of four developmental milestones (hatching, fourth larval stage (L4), adult stage/young adulthood, and the onset of reproduction/reproductive adulthood) was measured at four temperatures: $15^{\circ} \mathrm{C}, 20^{\circ} \mathrm{C}, 25^{\circ} \mathrm{C}$, and $30^{\circ} \mathrm{C}$. For synchronization, mid-stage embryos (blastula to 1.5 fold stage) were picked from plates cultured at $25^{\circ} \mathrm{C}$ to new plates and then shifted to the given rearing temperature. Plates were then monitored hourly (for hatching) and then daily (for L4, young adulthood, and reproductive adulthood) for the onset of developmental milestones. Male tail and female/hermaphrodite vulva morphologies were used to define L4 and young adult stages. The onset of reproduction was scored only among females and hermaphrodites by the presence of embryos in the uterus. Plates were assayed until the number of individuals at or older than a given milestone did not increase for two hours or days. Animals who failed to reach a given milestone were not used for subsequent analysis. For analysis, animals were plotted by their developmental status (" 0 " = yet to reach milestone; " $1 "$ = reached milestone) over time and logistic regression was used to estimate the median time to a given event via the "glm" function (using a binomial distribution) in the R statistical language. This models approach was used for hypothesis testing and for calculating 95\% confidence intervals (see Additional file 4; data are available in Additional files 5 and 6).

\section{Lifespan}

Synchronized animals were generated by allowing gravid females/hermaphrodites (20 C. elegans hermaphrodites or C. elegans fog-2(q71) pseudo-females per plate; about 100 C. inopinata females per plate) to lay for 2-3 hours. After a few days, synchronized L4 virgin females/hermaphrodites were moved to new plates, with about 30 nematodes per plate. All animals were transferred every day for the first 4-5 days of adulthood as hermaphrodites reproduced. Subsequently, animals were scored every 1-3 days as either living or dead up until the point that all animals had died. All measurements were performed at $25^{\circ} \mathrm{C}$. The number of days alive after egg-laying was taken as the measure of total lifespan. Lifespan and longevity studies in C. elegans are often concerned with the basis of aging, which is generally thought to largely occur in adulthood after developmental growth [84]. Thus we report here both total lifespan (starting at embryogenesis) and adult lifespan (starting at the onset of maturation). As C. inopinata and C. elegans display different rates of developmental growth, this also allows a comparison of the rate of aging in adults that accounts for this difference. Adult lifespan was taken as the total lifespan minus two $(C$. elegans) or four (C. inopinata) days, as C. inopinata develops at about half the rate as C. elegans. Statistical analyses were performed as in [38], with the survival package for the $\mathrm{R}$ statistical language being used to generate survivorship curves and the coxme package being used to generate Cox proportional hazard models and perform hypothesis tests (see Additional file 4; data are available in Additional file 7).

\section{Fecundity}

Daily offspring production was measured following overnight mating and under continuous exposure to 
males. For all observations, L4 C. inopinata NKZ2 and C. elegans fog-2(q71) animals raised at $25^{\circ} \mathrm{C}$ were isolated and raised for one (C. elegans) or two (C. inopinata) days to adulthood (see above). For overnight mating, single adult females/pseudo-females were shifted to the given experimental rearing temperature and mated with six males overnight. Brood sizes were measured at $15^{\circ} \mathrm{C}$, $20^{\circ} \mathrm{C}, 25^{\circ} \mathrm{C}$, and $30^{\circ} \mathrm{C}$. The next day males were removed. Every day, embryos and larvae were counted, and egg-laying females were moved to new plates. New progeny were scored until females stopped laying for at least one (C. elegans) or two (C. inopinata) consecutive days. Continuous mating conditions were similar, except that single females were always in the presence of six males. Males that crawled up the side of the plate or otherwise died before the female stopped laying embryos were replaced with young adult males. The continuous mating observations were performed at $25^{\circ} \mathrm{C}$. The instantaneous rate of natural increase [1] was calculated in Python as in [85] using life tables for $C$. elegans and C. inopinata constructed from the viability, fecundity, and lifespan data developed here (see Additional file 8; data are available in Additional files $9,10,11$ and 12).

\section{Embryo-to-adult viability}

Nematode embryos were synchronized by allowing gravid females/hermaphrodites (20 C. elegans hermaphrodites or C. elegans fog-2(q71) pseudo-females per plate; about $100 \mathrm{C}$. inopinata females per plate) to lay for 2-3 hours. After the parents were removed, the number of embryos per plate were counted, and the plates were shifted to their respective rearing temperatures $\left(15^{\circ} \mathrm{C}\right.$, $20^{\circ} \mathrm{C}, 25^{\circ} \mathrm{C}$, or $30^{\circ} \mathrm{C}$ ). $\mathrm{L} 4$ and adult worms were counted 4-5 days later. This fraction of mature worms/initial worm embryos was reported as the viability. Data are available in Additional file 13.

\section{Intersection of WormBase phenotypes related to life history traits among C. elegans protein-coding genes} Functional annotations for all C. elegans protein-coding genes were retrieved using the simplemine tool in WormBase (http://www.wormbase.org/tools/mine/simplemine.cgi, link labeled "query all C. elegans"; Additional file 14). Genes with mutant or RNAi phenotypes "long," "slow growth," "extended life span," and "reduced brood size" were extracted, and a spreadsheet denoting the intersection of these four phenotypes for every gene that included at least one of these phenotypes was created with Linux (see Additional file 15 for software) and Perl (https://github.com/religa/ stats/blob/master/merge) tools (see Additional files 14 and 15 for data). The $U p S e t R$ package [86] was used to make Fig. 6 with this data (Additional file 4).

\section{Additional files}

Additional file 1: Figure S1. Total lifespan models with 95\% confidence intervals. Figure S2. Adult lifespan models with 95\% confidence intervals. Figure S3. Patterns of failed crosses across mating conditions and temperatures. Figure S4. C. inopinata has lower brood sizes than $C$. elegans (fog-2) in continuous mating conditions after removing failed crosses. Figure S5. Comparison of intra- and interspecific variation in Caenorhabditis fecundity in four recent studies (with data colored by publication). Figure S6. Comparison of intra- and interspecific variation in Caenorhabditis fecundity in four recent studies (with data colored by strain locality). Figure S7. Comparison of intra- and interspecific variation in Caenorhabditis age of maturation in three studies (with data colored by publication). Figure S8. Comparison of intra- and interspecific variation in Caenorhabditis age of maturation in three studies (with data colored by strain locality). Table $\mathbf{S 1}$. Estimates of median time of developmental events. (PDF $682 \mathrm{~kb}$ )

Additional file 2: Fecundity_metadata.tsv. Fecundity metadata collected for Additional file 1: Figure S5-S6. (TSV $42 \mathrm{~kb}$ )

Additional file 3: Developmental_timing_metadata.tsv. Developmental timing metadata collected for Additional file 1: Figure S7-S8. (TSV 6 kb)

Additional file 4: Models_hypothesis_tests.R. Software for generating models and statistics. (R $52 \mathrm{~kb}$ )

Additional file 5: Hatch_time_data.tsv. Developmental timing data for hatching. (TSV $7 \mathrm{~kb}$ )

Additional file 6: Postembryonic_milestone_time_data.tsv. Developmental timing data for postembryonic milestones. (TSV $37 \mathrm{~kb}$ )

Additional file 7: Lifespan_data.tsv. Lifespan data. (TSV 42 kb)

Additional file 8: Estimate_r.py. Software for estimating the rate of population increase. (PY $14 \mathrm{~kb}$ )

Additional file 9: Reproductive_duration_data.tsv. Reproductive duration data. (TSV $15 \mathrm{~kb}$ )

Additional file 10: Fecundity_lifetime_access_data.tsv. Fecundity with lifetime access to males data. (TSV $2 \mathrm{~kb}$ )

Additional file 11: Fecundity_overnight_mating_data.tsv. Fecundity with one overnight mating data. (TSV $2 \mathrm{~kb}$ )

Additional file 12: Life_tables.tsv. Data used for estimating the rate of population increase. (TSV $14 \mathrm{~kb}$ )

Additional file 13: Viability_data.tsv. Viability data. (TSV 1 kb)

Additional file 14: Wormbse_simplemine.txt. Data retrieved from WormBase for generating upset_plot_input.tsv (Additional file 16). (TXT 5290 kb)

Additional file 15: Wormbase_phenotype_intersections.sh. Software for generating phenotype intersection UpSet plot data from a WormBase Simplemine tab-delimited file. (SH 6 kb)

Additional file 16: Upset_plot_input.tsv. Data for generating an UpSet plot as in Figure. (TSV $453 \mathrm{~kb}$ )

\section{Abbreviations}

Cl: Confidence interval; Fog: Feminization of germline; GLM: Generalized linear model; L4: Fourth larval stage; LRT: Likelihood ratio test

\section{Acknowledgements}

We thank two anonymous reviewers whose helpful comments improved this manuscript.

\section{Funding}

This work was supported by funding from the National Institutes of Health to GCW (5F32GM115209-03) and PCP (R01 GM-102511; R01 AG049396). This funding body had no role in the design of the study; the collection, analysis, 
and interpretation of data; and in the drafting and revising of the manuscript.

\section{Availability of data and materials}

All data generated or analyzed during this study (Additional files 5, 6, 7, 9, 10, $11,13,12,14,14,15$, and 16), as well as software used to analyze this data (Additional files 4, 8 and 15), are included in this published article.

\section{Authors' contributions}

GCW and PCP designed the experiments; GCW and EJ performed the experiments; GCW analyzed the results; GCW and PCP wrote the paper. All authors read and approved the final manuscript.

\section{Ethics approval and consent to participate}

Not applicable.

\section{Consent for publication}

Not applicable.

\section{Competing interests}

The authors declare that they have no competing interests.

\section{Publisher's Note}

Springer Nature remains neutral with regard to jurisdictional claims in published maps and institutional affiliations.

Received: 3 October 2018 Accepted: 13 February 2019

Published online: 11 March 2019

\section{References}

1. Stearns SC. The evolution of life histories, vol. 249. Oxford: Oxford University Press; 1992.

2. Roff DA. Life history evolution; 2002

3. Hamilton WD. The moulding of senescence by natural selection. J Theor Biol. 1966:12(1):12-45.

4. Emlen JM. Age specificity and ecological theory. Ecology. 1970;51(4): 588-601.

5. Charlesworth B. Evolution in age-structured populations. Cambridge: Cambridge University Press; 1980.

6. Clutton-Brock TH, Guinness FE, Albon SD. Red deer: behavior and ecology of two sexes. Chicago: University of Chicago press; 1982.

7. Reznick D. Costs of reproduction: an evaluation of the empirical evidence. Oikos. 1985;44:257-67.

8. Luckinbill LS, Arking R, Clare MJ, Cirocco WC, Buck SA. Selection for delayed senescence in Drosophila melanogaster. Evolution. 1984;38(5):996-1003.

9. Reznick D. The structure of guppy life histories: the tradeoff between growth and reproduction. Ecology. 1983;64(4):862-73.

10. Robinson B, Doyle R. Trade-off between male reproduction (amplexus) and growth in the amphipod Gammarus lawrencianus. Biol Bull. 1985; 168(3):482-8

11. Ghalambor CK, Reznick DN, Walker JA. Constraints on adaptive evolution: the functional trade-off between reproduction and fast-start swimming performance in the Trinidadian guppy (Poecilia reticulata). Am Nat. 2004; 164(1):38-50.

12. Fleming IA, Gross MR. Latitudinal clines: a trade-off between egg number and size in Pacific salmon. Ecology. 1990;71(1):1-11.

13. Sadras VO. Evolutionary aspects of the trade-off between seed size and number in crops. Field Crop Res. 2007;100(2):125-38.

14. McMahon TA, Bonner JT. On size and life. New York: Scientific American Library; 1983.

15. Calder WA. Size, function, and life history. Cambridge: Harvard University Press; 1984.

16. Schmidt-Nielsen K. Scaling: why is animal size so important? Cambridge Cambrige University Press; 1984.

17. Peters RH. The ecological implications of body size, vol. 2. Cambridge: Cambridge University Press; 1986

18. Stanley SM. An explanation for Cope's rule. Evolution. 1973;27(1):1-26

19. Sibly RM, Calow P. Physiological ecology of animals. 19. Oxford: Blackwell Scientific Publications; 1986.

20. Blanckenhorn WU. The evolution of body size: what keeps organisms small? Q Rev Biol. 2000;75(4):385-407.
21. Partridge $\mathrm{L}$, Harvey $\mathrm{PH}$. The ecological context of life history evolution Science. 1988:241(4872):1449-55.

22. >Reznick D, Endler JA. The impact of predation on life history evolution in Trinidadian guppies (Poecilia reticulata). Evolution. 1982;36(1):160-77.

23. Corsi AK, Wightman B, Chalfie M. A transparent window into biology: a primer on Caenorhabditis elegans. Genetics. 2015;200(2):387-407.

24. Keightley PD, Davies EK, Peters AD, Shaw RG. Properties of ethylmethane sulfonate-induced mutations affecting life-history traits in Caenorhabditis elegans and inferences about bivariate distributions of mutation effects. Genetics. 2000;156(1):143-54.

25. Azevedo RB, Keightley PD, Laurén-Määttä C, Vassilieva LL, Lynch M, Lero AM. Spontaneous mutational variation for body size in Caenorhabditis elegans. Genetics. 2002;162(2):755-65.

26. McCulloch D, Gems D. Body size, insulin/IGF signaling and aging in the nematode Caenorhabditis elegans. Exp Gerontol. 2003:38(1):129-36.

27. Estes S, Ajie BC, Lynch M, Phillips PC. Spontaneous mutational correlations for life-history, morphological and behavioral characters in Caenorhabditis elegans. Genetics. 2005;170(2):645-53.

28. Ostrow D, Phillips N, Avalos A, Blanton D, Boggs A, Keller T, et al. Mutational bias for body size in rhabditid nematodes. Genetics. 2007;176(3):1653-61.

29. Kammenga JE, Doroszuk A, Riksen JA, Hazendonk E, Spiridon L, Petrescu A-J, et al. A Caenorhabditis elegans wild type defies the temperature-size rule owing to a single nucleotide polymorphism in tra-3. PLoS Genet. 2007;3(3):e34

30. Anderson JL, Albergotti L, Proulx S, Peden C, Huey RB, Phillips PC. Thermal preference of Caenorhabditis elegans: a null model and empirical tests. J Exp Biol. 2007:210(17):3107-16.

31. Anderson JL, Albergotti L, Ellebracht B, Huey RB, Phillips PC. Does thermoregulatory behavior maximize reproductive fitness of natural isolates of Caenorhabditis elegans? BMC Evol Biol. 2011;11(1):157.

32. Anderson JL, Reynolds RM, Morran LT, Tolman-Thompson J, Phillips PC. Experimental evolution reveals antagonistic pleiotropy in reproductive timing but not life span in Caenorhabditis elegans. J Gerontol A Biol Sci Med Sci. 2011;66(12):1300-8

33. Frézal L, Félix MA. C. elegans outside the Petri dish. Elife. 2015:4:e05849.

34. Kanzaki N, Tsai IJ, Tanaka R, Hunt VL, Tsuyama K, Liu D, et al. Biology and genome of a newly discovered sibling species of Caenorhabditis elegans. Nat Commun. 2018:9:1-12. https://www.nature.com/articles/s41467-018-05712-5

35. Woodruff GC, Willis JH, Phillips PC. Dramatic evolution of body length due to post-embryonic changes in cell size in a newly discovered close relative of C. elegans. Evolution Letters. 2018;2:427-41.

36. Kiontke KC, Félix M-A, Ailion M, Rockman MV, Braendle C, Pénigault J-B, et al. A phylogeny and molecular barcodes for Caenorhabditis, with numerous new species from rotting fruits. BMC Evol Biol. 2011;11(1):339.

37. Woodruff GC, Phillips PC. Field studies reveal a close relative of $C$. elegans thrives in the fresh figs of Ficus septica and disperses on its Ceratosolen pollinating wasps. BMC Ecol. 2018;18:1-16. https://bmcecol.biomedcentral. com/articles/10.1186/s12898-018-0182-z

38. Lucanic M, Plummer WT, Chen E, Harke J, Foulger AC, Onken B, et al. Impact of genetic background and experimental reproducibility on identifying chemical compounds with robust longevity effects. Nat Commun. 2017:8:14256.

39. Snell TW, King CE. Lifespan and fecundity patterns in rotifers: the cost of reproduction. Evolution. 1977:31(4):882-90.

40. Cox RM, Calsbeek R. Severe costs of reproduction persist in Anolis lizards despite the evolution of a single-egg clutch. Evolution. 2010;64(5):1321-30.

41. Schedl T, Kimble J. fog-2, a germ-line-specific sex determination gene required for hermaphrodite spermatogenesis in Caenorhabditis elegans Genetics. 1988;119(1):43-61.

42. Palopoli MF, Peden C, Woo C, Akiha K, Ary M, Cruze L, et al. Natural and experimental evolution of sexual conflict within Caenorhabditis nematodes. BMC Evol Biol. 2015;15(1):93.

43. Diaz SA, Haydon DT, LindstrÖM J. Sperm-limited fecundity and polyandry-induced mortality in female nematodes Caenorhabditis remanei. Biol J Linn Soc. 2010;99(2):362-9.

44. Thomas CG, Woodruff GC, Haag ES. Causes and consequences of the evolution of reproductive mode in Caenorhabditis nematodes. Trends Genet. 2012;28(5):213-20.

45. Charlesworth B, Charlesworth D. Elements of evolutionary genetics, vol. 42 Greenwood Village, CO: Roberts and Company Publishers; 2010. 
46. Roff DA. The evolution of genetic correlations: an analysis of patterns. Evolution. 1996;50(4):1392-403

47. Lee RYN, Howe KL, Harris TW, Arnaboldi V, Cain S, Chan J, et al. WormBase 2017: molting into a new stage. Nucleic Acids Res. 2017;46(D1):D869-74

48. Schindelman G, Fernandes JS, Bastiani CA, Yook K, Sternberg PW. Worm Phenotype Ontology: integrating phenotype data within and beyond the C. elegans community. BMC bioinformatics. 2011;12(1):32.

49. Petersen $C$, Dirksen $P$, Schulenburg H. Why we need more ecology for genetic models such as C. Elegans. Trends Genet. 2015;31(3):120-7.

50. Bonner JT. Size and cycle. Princeton: Princeton University Pres; 1965

51. Cutter AD. Caenorhabditis evolution in the wild. BioEssays. 2015;37(9): 983-95

52. Janzen DH. How to be a fig. Annu Rev Ecol Syst. 1979;10(1):13-51.

53. Schulenburg H, Félix M-A. The natural biotic environment of Caenorhabditis elegans. Genetics. 2017;206(1):55-86.

54. Dirksen P, Marsh SA, Braker I, Heitland N, Wagner S, Nakad R, et al. The native microbiome of the nematode Caenorhabditis elegans: gateway to a new host-microbiome model. BMC Biol. 2016:14(1):1.

55. Samuel BS, Rowedder H, Braendle C, Félix M-A, Ruvkun G. Caenorhabditis elegans responses to bacteria from its natural habitats. Proc Natl Acad Sci U S A. 2016;113(27):E3941-9.

56. Morand S, Sorci G. Determinants of life-history evolution in nematodes. Parasitol Today. 1998;14(5):193-6.

57. Morand S. Life-history traits in parasitic nematodes: a comparative approach for the search of invariants. Funct Ecol. 1996;10:210-8.

58. Skorping A, Read AF, Keymer AE. Life history covariation in intestinal nematodes of mammals. Oikos. 1991;60:365-72.

59. Herre EA, Jandér KC, Machado CA. Evolutionary ecology of figs and their associates: recent progress and outstanding puzzles. Annu Rev Ecol Evol Syst. 2008;39:439-58.

60. Konno K. Plant latex and other exudates as plant defense systems: roles of various defense chemicals and proteins contained therein. Phytochemistry. 2011;72(13):1510-30

61. Proffit M, Schatz B, Bessière J-M, Chen C, Soler C, Hossaert-McKey M. Signalling receptivity: comparison of the emission of volatile compounds by figs of Ficus hispida before, during and after the phase of receptivity to pollinators. Symbiosis (Rehovot). 2008:45(1):15.

62. Nurse P. Genetic control of cell size at cell division in yeast. Nature. 1975; 256(5518):547

63. Stocker H, Hafen E. Genetic control of cell size. Curr Opin Genet Dev. 2000; 10(5):529-35.

64. Salomon MP, Ostrow D, Phillips N, Blanton D, Bour W, Keller TE, et al. Comparing mutational and standing genetic variability for fitness and size in Caenorhabditis briggsae and C. Elegans. Genetics. 2009;183(2):685-92.

65. Honěk A. Intraspecific variation in body size and fecundity in insects: a general relationship. Oikos. 1993;66:483-92.

66. Woodruff GC, Eke O, Baird SE, Félix M-A, Haag ES. Insights into species divergence and the evolution of hermaphroditism from fertile interspecies hybrids of Caenorhabditis nematodes. Genetics. 2010;186(3):997-1012.

67. Dey A, Jeon Y, Wang G-X, Cutter AD. Global population genetic structure of Caenorhabditis remanei reveals incipient speciation. Genetics. 2012;191(4): 1257-69.

68. Dey A, Chan CK, Thomas CG, Cutter AD. Molecular hyperdiversity defines populations of the nematode Caenorhabditis brenneri. Proc Natl Acad Sci U S A. 2013;110(27):11056-60

69. Bundus JD, Alaei R, Cutter AD. Gametic selection, developmental trajectories, and extrinsic heterogeneity in Haldane's rule. Evolution. 2015; 69(8):2005-17

70. Williams GC. Pleiotropy, natural selection, and the evolution of senescence. Evolution. 1957;11:398-411.

71. Sudhaus W, Kiontke K. Comparison of the cryptic nematode species Caenorhabditis brenneri sp. n. and C. remanei (Nematoda: Rhabditidae) with the stem species pattern of the Caenorhabditis Elegans group. Zootaxa. 2007; 1456:45-62.

72. Félix M-A, Braendle $C$, Cutter AD. A streamlined system for species diagnosis in Caenorhabditis (Nematoda: Rhabditidae) with name designations for 15 distinct biological species. PLoS One. 2014;9(4):e94723.

73. Ebel ER, Phillips PC. Intrinsic differences between males and females determine sex-specific consequences of inbreeding. BMC Evol Biol. 2016; 16(1):36.
74. Sterken MG, Snoek LB, Kammenga JE, Andersen EC. The laboratory domestication of Caenorhabditis elegans. Trends Genet. 2015;31(5):224-31.

75. Diaz SA, Lindström J, Haydon DT. Basic demography of Caenorhabditis remanei cultured under standard laboratory conditions. J Nematol. 2008; 40(3):167.

76. Dolgin ES, Charlesworth B, Baird SE, Cutter AD. Inbreeding and outbreeding depression in Caenorhabditis nematodes. Evolution. 2007;61(6):1339-52.

77. Barrière A, Yang S-P, Pekarek E, Thomas CG, Haag ES, Ruvinsky I. Detecting heterozygosity in shotgun genome assemblies: lessons from obligately outcrossing nematodes. Genome Res. 2009;19:470-80.

78. Dolgin E, Felix M, Cutter A. Hakuna Nematoda: genetic and phenotypic diversity in African isolates of Caenorhabditis elegans and C. briggsae. Heredity. 2008;100(3):304.

79. Thomas CG, Wang W, Jovelin R, Ghosh R, Lomasko T, Trinh Q, et al. Fullgenome evolutionary histories of selfing, splitting, and selection in Caenorhabditis. Genome Res. 2015;25(5):667-78.

80. Prasad A, Croydon-Sugarman MJ, Murray RL, Cutter AD. Temperaturedependent fecundity associates with latitude in Caenorhabditis briggsae. Evolution. 2011:65(1):52-63.

81. Kozlowska JL, Ahmad AR, Jahesh E, Cutter AD. Genetic variation for postzygotic reproductive isolation between Caenorhabditis briggsae and Caenorhabditis sp. 9. Evolution. 2012;66(4):1180-95.

82. Poullet N, Vielle A, Gimond C, Ferrari C, Braendle C. Evolutionarily divergent thermal sensitivity of germline development and fertility in hermaphroditic Caenorhabditis nematodes. Evol Dev. 2015;17(6):380-97.

83. Ferrari C, Salle R, Callemeyn-Torre N, Jovelin R, Cutter AD, Braendle C. Ephemeral-habitat colonization and neotropical species richness of Caenorhabditis nematodes. BMC Ecol. 2017;17(1):43.

84. Collins JJ, et al. The measurement and analysis of age-related changes in Caenorhabditis elegans (December 7, 2007), WormBook, ed. The C. elegans Research Community, WormBook, https://doi.org/10.1895/wormbook.1.137. 1, http://www.wormbook.org.

85. Hill C. Learning scientific programming with python. Cambridge: Cambridge University Press; 2016.

86. Conway JR, Lex A, Gehlenborg N. UpSetR: an R package for the visualization of intersecting sets and their properties. Bioinformatics. 2017;33(18):2938-40.
Ready to submit your research? Choose BMC and benefit from:

- fast, convenient online submission

- thorough peer review by experienced researchers in your field

- rapid publication on acceptance

- support for research data, including large and complex data types

- gold Open Access which fosters wider collaboration and increased citations

- maximum visibility for your research: over 100M website views per year

At BMC, research is always in progress.

Learn more biomedcentral.com/submissions 